Bundesgesundheitsbl 2020 - 63:997-1003 https://doi.org/10.1007/s00103-020-03184-x Online publiziert: 10. Juli 2020

(c) Der/die Autor(en) 2020

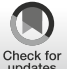

Susanne Moebus ${ }^{1}$. Dietwald Gruehn² . Jonas Poppen ${ }^{1} \cdot$ Robynne Sutcliffe ${ }^{1}$ • Timo Haselhoff' · Bryce Lawrence ${ }^{2}$

'Institut für Urban Public Health, Universitätsmedizin, Universität Duisburg-Essen, Essen, Deutschland

${ }^{2}$ Fakultät Raumplanung, Lehrstuhl Landschaftsökologie und Landschaftsplanung, TU Dortmund, Dortmund, Deutschland

\title{
Akustische Qualität und Stadtgesundheit - Mehr als nur Lärm und Stille
}

bedeutet Schallschutz - umgangssprachlich und in vielen Rechtsverordnungen noch häufig als „Lärmschutz“ bezeichnet - in einer urbanen Umwelt? Und was ist das Pendant von Lärm: Ruhe oder gar Stille? Ist Stille überhaupt möglich in einer verdichteten städtischen Umwelt und ist Stille als alleiniges Ziel überhaupt erstrebenswert? Ist der Fokus auf Lärm ausreichend, um eine gesundheitsförderliche akustische Qualität in Städten zu erreichen?

Der vorliegende Beitrag hat zum Ziel, die akustische Qualität in städtischen Räumen als einen bislang vernachlässigten Aspekt der gesundheitsförderlichen Stadtentwicklung in Public Health einzuführen. Dazu erfolgt zunächst ein kurzer Überblick zu den Begriffen „Lärm“ und „Geräusch“. Anschließend werden 2 Ansätze der Forschung zu „Klanglandschaften“ (Soundscapes) beschrieben, um abschließend Ziele und Methodik eines Pilotprojektes zum Thema gesundheitsförderliche akustische Qualität urbaner Räume vorzustellen.

\section{Lärm und Geräusche}

Menschen bewegen sich ständig in akustischen Räumen, die durch Lautstärke als auch die Zusammensetzung von Geräuschen charakterisiert sind. Lärm ist dabei eines der bekanntesten Phänomene in akustischen Räumen und ein weitverbreiteter urbaner Stressor. Lärm mindert Wohn- und Schlafqualität, fördert Aggressionen, lenkt ab, vermindert die akustische Variation einer Umgebung und erschwert die Kommunikation und Konzentration [4], soziale Interaktion und soziales Engagement werden eingeschränkt [5]. So waren 2012 allein in den europäischen Ländern geschätzte 125 Mio. Menschen Straßenverkehrslärm $\geq 55 \mathrm{~dB}(\mathrm{~A})$ ausgesetzt [6]. Lärm ist als Gesundheitsrisiko ein weltweit anerkanntes Problem, so wird vor allem (chronischer) Verkehrslärm mit erhöhtem Blutdruck [7-9], Myokardinfarkt [10, 11], Schlaganfall [12], Arteriosklerose [13] und Diabetes mellitus Typ 2 [14] assoziiert. Schlafstörungen durch Lärm werden mit Depressionen assoziiert $[15,16]$ und Stressreaktionen können psychische Probleme fördern [17, 18].

Trotz der häufig beschriebenen Assoziationen von Lärm und Krankheit wird Lärm erstaunlich unterschiedlich umschrieben: Das Bundesministerium für Umwelt, Naturschutz und nukleare Sicherheit (BMU) etwa definiert Lärm als jedes unerwünschte laute Geräusch und bezeichnet Lärm als subjektiv geprägten Begriff [19]. Die Bundeszentrale für gesundheitliche Aufklärung (BZgA) beschreibt in ihren Unterrichtsmaterialien $\mathrm{zu}$ Basisthemen der Gesundheitserziehung und -förderung für die 5. bis 10. Klasse Lärm als „Schall, der als störend empfunden wird (subjektives Merkmal) und der gesundheitsgefährdend oder gesundheitsschädigend ist (objektives Merkmal)“ [20]. Diesen Auffassungen ist gemein, dass Lärm als Folge einer subjektiven Einschätzung verstanden wird. Andere Auffassungen verstehen „störend“ und „unerwünscht“ 
aber offenbar nicht als ein unverzichtbares Element der Definition, sondern lediglich als einen weiteren Aspekt [21, 22]. Nach der DIN 1320 wird Lärm definiert als „Hörschall, der zu Störungen, Belästigungen, Beeinträchtigungen oder Schäden führen kann“" [23]. In der technischen Anleitung zum Schutz gegen Lärm (TA Lärm) der Bundesregierung wird Lärm als „schädliche Umwelteinwirkungen durch Geräusche“ beschrieben, die wiederum als „Geräuschimmissionen, die nach Art, Ausmaß oder Dauer geeignet sind, Gefahren, erhebliche Nachteile oder erhebliche Belästigungen für die Allgemeinheit oder die Nachbarschaft herbeizuführen“, definiert werden [24]. Oder auch: Lärm wird als „derjenige Schall bezeichnet, der das körperliche, seelische und soziale Wohlbefinden von Menschen beeinträchtigt “ [25]. Dagegen stehen Auffassungen, die gänzlich ohne eine subjektive Komponente auskommen: In der Lärm- und VibrationsArbeitsschutzverordnung ist Lärm jeder Schall, der zu einer Beeinträchtigung des Hörvermögens oder der zu einer sonstigen mittelbaren oder unmittelbaren Gefährdung von Sicherheit und Gesundheit der Beschäftigten führen kann [26]. Auch das BMU ist trotz seiner Einschätzung, dass der Begriff Lärm subjektiv geprägt ist, der Auffassung, „Lärm kann man mit geeigneten Geräten messen“" [27].

Nicht zuletzt sei hier das häufig zitierte Bonmot von Tucholsky erwähnt, der Lärm als „,das Geräusch der anderen“ [28] treffend beschrieb. Aber was ist eigentlich ein Geräusch? Die denkbar simpelste Erklärung wäre, dass alles, was wir hören, Geräusche sind. Es scheint aber komplizierter. Einheitliche Beschreibungen sind ähnlich wie beim Lärm schwer zu finden; so sind Geräusche ein akustisch wahrnehmbarer, unspezifischer Vorgang, ein hörbares Ereignis [29], ein aus Tönen wechselnder Höhe, Stärke und Klangfarbe zusammengesetzter Schall [30], ein Sammelbegriff für alle Hörempfindungen, die nicht als Klang, Tongemisch, Zusammenklang oder Klanggemisch bezeichnet werden können bzw. Schwingungsvorgänge, die in der Regel nicht periodisch verlaufen und sich in ihrer Struktur zeitlich ändern können [31], oder auch als Schall, der nicht vorwiegend zur Übertragung von Information dient [23]. Und als letztes Beispiel für die Erklärung für Geräusche: Ein Geräusch ist demnach (auch) ein akustisches Signal mit zahlreichen Teilfrequenzen, zwischen denen kein gesetzmäßiger Zusammenhang besteht, wie dies z. B. beim Klang der Fall ist [2, 2, 32]. Es wird deutlich, dass bei den aufgeführten Beschreibungen von Geräuschen wiederum viele Begrifflichkeiten aus unterschiedlichen Kategorien Verwendung finden, wie Schwingungsvorgänge, Frequenz und Pegelhöhe, akustisches Signal, Schallsignale, zusammengesetzter Schall, Töne, Klang, akustische Wahrnehmung, Hörempfindung, die auf verschiedene methodologische Ansätze hinweisen.

\section{Mehr als Lärm: Geräusche, akustische Qualität und Public Health}

Die Lärmwirkungsforschung und der umweltbezogene Gesundheitsschutz beruhen auf einem etablierten schallpegelbasierten Modellansatz und resp. Dosis-Wirkungs-Beziehungen [33]. So wird z.B. die Höhe des Schallpegels von lärmerzeugenden Schallquellen geschätzt, die als jährliche äquivalente Schalldruckpegel ( $\mathrm{L}_{\text {den }}$ und $\mathrm{L}_{\text {night }}$ ) häufig verwendet werden. Die Eingrenzung der Geräusche auf den Schalldruckpegel führt $\mathrm{zu}$ Lösungsstrategien, die auf Lärmminderung abzielen. Für diese Maßnahmen steht europaweit bereits eine Reihe von Verordnungen, Richtlinien (Umgebungslärmrichtlinie), Richtwerten und Aktionsplänen zur Verfügung.

Im Rahmen des notwendigen Lärmschutzes werden allerdings widersprüchliche oder auch unerwartete Ergebnisse beobachtet. Deshalb sollen im Folgenden kurz einige Argumente für eine Blickfelderweiterung des traditionellen, schallpegelbasierten Modellansatzes skizziert werden. Ein Argument ist die immer wieder festgestellte Diskrepanz zwischen Geräuschen unterhalb eines als gesundheitsschädlich festgelegten Dauerschallpegels und Belästigung durch Lärm [34]. So kann nach Guski nur rund ein Drittel der Varianz von Belästigungsurteilen durch akustische Kenn- größen erklärt werden [35]. Ein weiteres Argument ist, dass die bisherige Fokussierung auf die alleinige Senkung der Geräuschpegel nicht zwingend auch die Wirkung von Lärm reduziert und sich damit der Erfolg einer Maßnahme nicht unbedingt direkt aus einer ExpositionsWirkungs-Beziehung ableiten lässt [36]. Das heißt auch, dass bauliche Lärmminderungsmaßnahmen nicht immer den erwarteten (berechneten) Erfolg erzielen [36].

Die Rolle räumlicher Bedingungen wird häufig unterschätzt, z. B. können durch Lärmschutzwände neue ungünstige Resonanzräume geschaffen werden [37]. Ein Weniger an Lärm bedeutet auch nicht unbedingt eine bessere akustische Qualität [38]. Im Gegenteil, Schallschutzfenster und Lärmschutzmauern isolieren Bewohner*innen von ihrem Umfeld, verbunden mit einem Rückzug aus dem öffentlichen Raum. Maag [39] skizziert es folgend: „Schallschutzbauwerke isolieren vor allem visuell, grenzen aus und erschweren die Durchlässigkeit ... sie machen auf Lärm aufmerksam ... und ... sie ergeben akustisch oft keinen räumlich zusammenhängenden Sinn. ... Folgen wir dem Ideal der Lärmbekämpfung blindlings, isolieren wir uns zusehends und akzeptieren notgedrungen immer kleinere Hörräume. Zusammenhängende Stadträume zerfallen in einzelne akustische Fragmente. Der öffentliche Raum, den wir selber hören, wird immer kleiner ..." [39]. Solche „hörblind“ [40] ${ }^{1}$ gestalteten Räume können die Menschen von ihrer Umwelt isolieren.

Mittlerweile wird zudem bezweifelt, dass die technischen Entwicklungen von Lärmminderungsmaßnahmen durch die

\footnotetext{
1 Unter "hörblind" verstehen Bosshard und Maag: „Halten wir uns dauernd in lärmbelasteten Räumen auf, hören wir lediglich die uns unmittelbar betreffenden Signale und kaum mehr Rauminformationen. Wir filtern die meisten Klänge und Geräusche weg und trauen dem Lärm keinerlei Qualitäten mehr zu. Wir vergessen zunächst den akustischen Tiefenraum, dann sogar den gesamten klingenden Umraum. Wir sind damit nicht taub, sondern hörblind. Unsere Fähigkeit, akustisch wahrgenommene Impulse aus der Umwelt zu deuten, nimmt ab und uns fehlen zunehmend die Worte für die meisten klingenden Erscheinungen des Raumes" [40, S. 106].
} 
zunehmende Urbanisierung bzw. Verdichtung der Städte und die damit auch steigende Mobilität ausreichen werden [41]. Weiterhin wird zwar die Bedeutung ruhiger Orte international anerkannt, aber bisherige Ansätze reichen nicht aus, um gute ruhige Gebiete zu definieren bzw. methodisch zu erfassen [42]. Dies ist von zentraler Bedeutung und ein häufig vernachlässigtes Problem.

Durch die Verdichtung der Städte kann die räumliche Variation zwischen gewollten und ungewollten Klängen abnehmen oder sogar verschwinden [43]. Folglich werden urbane Räume, in denen sich Menschen vorübergehend von Stressfaktoren wie Lärm zurückziehen können, immer knapper werden. Ohne akzeptierte Definitionen und Messmethodiken, die gewährleisten, dass der Wert dieser Räume überhaupt erkannt wird, werden solche städtischen Räume kaum erhalten oder neu geschaffen werden können. Diese Sichtweise steht im Einklang mit den Empfehlungen der Richtlinie über Umgebungslärm, die Aktionspläne erfordern, die darauf abzielen, ruhige Gebiete in öffentlichen Räumen $\mathrm{zu}$ identifizieren und zu erhalten [44].

Zusammenfassend lässt sich festhalten, dass Schallschutz und Lärmminderungsmaßnahmen derzeit wichtige Elemente des gesundheitsbezogenen Umweltschutzes sind. Weniger Lärm bzw. eine alleinige Reduzierung der Geräuschpegel bedeutet aber nicht zwangsläufig eine gesundheitserhaltende oder -förderliche Klangqualität (Übersicht bei [45]). Deshalb sind weitere Ansätze zur Reduzierung des Lärms und zur Verbesserung der akustischen Umwelt notwendig [46]. Es braucht u. a. Indikatoren und Metriken, die Schalldruckpegel nicht nur von lärmerzeugenden Quellen schätzen, sondern eine gute akustische Qualität, d.h. urbane Klangqualität, indizieren können. Zusätzliche Kriterien müssen entwickelt werden, um die akustische Qualität in Städten in ihrer gänzlichen Breite zu erfassen, zu erhalten und zu erhöhen. Wissen und Forschung zu urbaner akustischer Qualität, insbesondere mit Blick auf Gesundheit, befinden sich noch in den Anfängen. Ungeklärt ist u.a., was eine gute akustische Qualität oder auch Klangqualität in Bezug auf urbane Räu-

Bundesgesundheitsbl 2020 -63:997-1003 https://doi.org/10.1007/s00103-020-03184-x (c) Der/die Autor(en) 2020

S. Moebus · D. Gruehn · J. Poppen · R. Sutcliffe · T. Haselhoff · B. Lawrence

\section{Akustische Qualität und Stadtgesundheit - Mehr als nur Lärm und Stille}

\section{Zusammenfassung}

Neue Ansätze in der Stadtentwicklung werden gefordert, um Städte in nachhaltige Orte zu transformieren. Das erfordert u. a. eine Verdichtung der Städte, die kaum denkbar ist ohne eine Zunahme der Geräuschentwicklung - meist in Form von Lärm. Um eine Akzeptanz für die Verdichtung zu erreichen, sind qualitätsvolle, gesundheitsförderliche akustische städtische Räume erforderlich. Hierfür bedarf es Ansätze, die über reine Lärmminderungsmaßnahmen hinausgehen. Ausgehend von der Frage: „Was hält gesund?", ist eine Perspektive, die auf eine gesundheitsförderliche städtische Klangqualität bzw. auf eine positiv bewertete akustische Umgebung zielt, erforderlich. Geräusche können als gestaltbare Qualität in urbanen Räumen als eine wichtige Ressource für eine gesundheitsförderliche Stadt verstanden werden. Hierfür werden über die schallpegelbasierten Indizes hinaus weitere Metriken benötigt, die eine gute urbane Klangqualität indizieren können. Wissen, Forschung und Anwendung zu urbaner akustischer Qualität, insbesondere mit Blick auf Gesundheit, befinden sich noch in den Anfängen. Für Urban Public Health schlagen wir eine Perspektiverweiterung durch die Verknüpfung und systematische Anwendung von zwei verschiedenen Soundscape-Ansätzen vor. Der vorliegende Beitrag gibt einen kurzen Überblick zu den Begriffen "Lärm" und "Geräusche", stellt zwei Ansätze der Soundscape-Forschung vor und legt anschließend Ziele und Methodik des Pilotprojektes SALVE (,akustische Qualität und Gesundheit in urbanen Räumen") zum Thema akustische Qualität in urbanen Räumen dar. Insgesamt wird ein Einblick in das neue Forschungs- und Praxisfeld von urbaner Klangqualität, Soundscapes und Stadtgesundheit gegeben.

Schlüsselwörter

Urban Public Health · StadtGesundheit . Stadtgeräusche · Klanglandschaft . Soundscapes

\section{Acoustic quality and urban health—more than just noise and silence}

\section{Abstract}

New approaches in urban development are required to transform cities into sustainable places. This demands a higher degree of urban density, which is hardly conceivable without an increase in sound - mostly in the form of noise. To achieve a high level of acceptance for densification, high-quality and acoustically pleasant urban spaces are essential. Noise reduction measures are necessary, but not sufficient. What is needed is a broadening of the perspective of noise. For urban public health we propose the soundscape approach from two different scientific disciplines. Here, sounds are qualities that can be designed in urban spaces and are an important resource for a healthy city. Linking knowledge about the acoustic environment with human perception will significantly improve our understanding of the relationships between urban acoustic environments, urban spatial contexts, and their effects on human health, both qualitatively and quantitatively. However, a systematic application of these approaches to urban soundscapes is still missing, as is the joint implementation of soundscape approaches in noise impact and urban public health research. This paper aims to introduce the terms sound and noise as well as two soundscape approaches. Subsequently, the aims and methods of the acoustic quality and health in urban environments (SALVE) pilot project are presented. The paper provides early insight into the new field of urban sound quality and soundscapes in the context of urban public health.

Keywords

Urban public health · Soundscapes · Urban acoustic quality · Urban environment · Sound 
me und Stadtentwicklung bedeutet, und noch weniger ist geklärt, welche Zusammenhänge zwischen der urbanen Klangqualität und der Gesundheit der Menschen bestehen.

\section{Urbane Klangqualität}

Ein vielversprechender Ansatz, um die offenen Fragen zu urbaner Klangqualität, Stadtentwicklung und Gesundheit systematisch zu analysieren, ermöglicht das Konzept der Soundscapes (Schallwelten, Klanglandschaften). Der Komponist Schafer hat neben dem Stadtplaner Southworth bereits in den 1960er-Jahren damit begonnen, die akustischen Umwelten als Teil der sinnlich erfassten Umwelt des Menschen zu systematisieren, und prägte dabei den Begriff Soundscape [47]. Klänge werden als gestaltbare Dimension von Schall definiert, die in Analogie zum Landschaftsbegriff quasi Klanglandschaften bilden. Der Forschungsgegenstand "Soundscapes“ nimmt bisher in den empirischen Wissenschaften wegen des zunächst eher ästhetisch-künstlerischen Schwerpunktes und der fehlenden quantifizierenden Methodik von Klanglandschaften eine eher randständige Rolle ein [4]. Der Ansatz eröffnet aber die Möglichkeit, die urbane Klangqualität einer Systematisierung für verschiedenste wissenschaftliche Disziplinen zugänglich zu machen, und kann damit für die notwendige Blickfelderweiterung interessant werden. Das Studium der urbanen Klangqualität für Public Health ist ein zukunftsweisendes, inter- und transdisziplinäres Forschungsfeld bei dem Bemühen, gesundheitsförderliche Ressourcen verdichteter Räume zu identifizieren und in die Praxis umzusetzen. Gründe hierfür sollen folgend kurz skizziert und anschließend ein erstes Pilotprojekt in Deutschland zu diesem Thema vorgestellt werden.

\section{Soundscapes als kognitiv- psychologisches Konstrukt}

Der oben beschriebene Soundscape-Ansatz hat sich in den letzten Jahren in 2 unterschiedliche Forschungs- und Handlungsfelder entwickelt, die entweder auf eine kognitiv-psychologische (auditori- sche Perzeption) oder physikalisch-akustische (ausgehend von der Messung von Schall bzw. Geräuschen) Perspektive fokussieren.

Die kognitiv-psychologische Dimension der Soundscapes zeigt eine große Nähe zur Psychoakustik. Bei diesem Soundscape-Ansatz wird explizit auf die Übersetzung des Begriffs "Soundscape“ in „Klanglandschaft“ verzichtet, da diese Übersetzung die Vielschichtigkeit des Begriffes „Soundscape“ nicht adäquat widerspiegelt [48]. Soundscapes existieren in diesem Ansatz erst durch die menschliche Wahrnehmung einer akustischen Umgebung. Die Grundannahme liegt darin, dass die Bedeutungen, die Menschen Geräuschen oder einem Expositionskontext geben, die damit verbundenen gesundheitlichen Auswirkungen stark beeinflussen [49]. So kann ein Geräusch lärmend, aber auch euphorisierend oder niederschmetternd sein (,Toooor"-Geschrei beim Fußballspiel). Daraus folgt, dass die Erfassung von Soundscapes nur sinnvoll ist, wenn (i) die Präferenz der Menschen für Soundscapes - und damit deren kognitive Wahrnehmung und Einschätzung - erfasst wird und (ii) der Kontext der Wahrnehmung einbezogen wird: Was an einem Ort von einer Person/ Personengruppe bevorzugt wird, kann an einem anderen Ort von dieser/diesen nicht bevorzugt werden.

Die Wahrnehmung der Soundscapes ist inter- und intraindividuell hoch variabel und wird neben den mentalen und physischen, sozialen oder kulturellen Faktoren auch durch Tages-, Wochenoder Jahreszeiten beeinflusst. So können Geräusche nicht nur als laut und leise wahrgenommen werden, sondern auch als schrill, angenehm, beruhigend, hämmernd, störend, monoton.

Für das kognitiv-psychologische Konstrukt von Soundscapes und die daraus folgenden methodischen Erfordernisse sind bereits Standards von der Internationalen Organisation für Normung (ISO) verabschiedet worden: (a) die International Organization for Standardization (ISO) zur Definition von Soundscapes [48] und (b) die Standardisierung der Methoden zur Datenerhebung [50]. Wenn, wie bei diesem Verständnis von Soundscapes, Lärm aus der Sicht der wahrnehmenden Personen analysiert und bewertet werden muss, sind mit diesem konzeptionellen Ansatz partizipative Verfahren, wie sie in der Stadtentwicklung und bei Public-HealthInterventionen vermehrt zum Einsatz kommen, naheliegend. Insbesondere Klangspaziergänge (Soundwalks), bei denen durch die gezielte Begehung zum Beispiel von Parks oder öffentlichen Plätzen die akustischen Umgebungen auch von Besucher*innen erfahren, bewertet und gemeinsam Lösungsstrategien entwickelt werden können, scheinen hierfür geeignet. Häufig geforderte Bedingungen für gelingende Partizipationsverfahren sind durch die vorhandene Expertise der Teilnehmenden quasi per definitionem erfüllt.

Ein Nachteil des Ansatzes ergibt sich allerdings zumindest aus empirischer Sicht. Da Soundscapes auf Grundlage der Wahrnehmung von Stimmungen, Erinnerungen und Vorstellungen gebildet werden - und streng genommen physikalische Schallmessungen keine Bedeutung haben -, ist eine entsprechend hohe inter- und intraindividuelle Variabilität zu erwarten. Damit stößt dieser Ansatz schnell an die Grenzen der Systematisierung und Dokumentation und erschwert so den empirischen $\mathrm{Zu}$ gang. Damit können sich auch Probleme für die politische Umsetzbarkeit ergeben. Auch wenn vereinzelt argumentiert wird, dass es zwischen den Menschen oft mehr Übereinstimmung als Nichtübereinstimmung bezüglich der bevorzugten Klanglandschaften eines beliebigen Ortes geben wird [51] und z.B. in der Stadtplanung ständig die Komplexität der gesellschaftlichen Bedürfnisse berücksichtigt werden muss [52], ist es aus Perspektive von Urban Public Health hilfreich, auch auf empirisch erzielte Ergebnisse für evidenzbasierte Lösungsansätze zurückgreifen zu können. Jedenfalls besteht bislang kein Konsens darüber, welche akustischen Indikatoren geeignet sind, gute urbane Klangqualität zu charakterisieren. Allenfalls deutet sich an, dass weitere akustische Indikatoren, wie z.B. der Hintergrundpegel (häufig gemessen als $\mathrm{L}_{A 50}, \mathrm{~L}_{A 90}$ und $\mathrm{L}_{A 95}$ ), die von den herkömmlichen $\mathrm{L}_{A e q}$-Metriken abweichen, wichtige Indikatoren sind, 
wenn auch noch immer nur mit mäßiger Zusammenhangsstärke zu angenehm empfundenen Geräuschen [43].

\section{Soundscapes als physikalisch- akustisches Konstrukt}

Die physikalisch-akustische Perspektive von Soundscapes hat ihre fachliche Nähe zur Soundscape Ecology, Bioakustik und Landschaftsökologie. Soundscapes werden hier definiert als die Gesamtheit der Klänge, die von einer Landschaft ausgehen und akustische Muster über räumliche und zeitliche Skalen erzeugen [53]. Dieser konzeptionelle Rahmen befasst sich mit der räumlichen und zeitlichen Heterogenität von Klängen in Landschaften. 3 grundlegende Geräuschquellen werden definiert, die durch Schalldruckpegel, Frequenz, Zeit und Raum bestimmt werden:

1. Geophonies: nicht-biologische Geräusche, wie Wind, Wasser, Donner;

2. Biophonies: Geräusche von nichtmenschlichen Organismen wie Kühen, Hunden, Vögeln und

3. Anthrophonies: alle menschlich erzeugten Geräusche, wie menschliche Stimmen, Musik, Maschinen, Autos.

Soundscapes existieren hier somit unabhängig von der Wahrnehmung der Menschen [54]. Die Frage, ob ein Klang, der von keinem Menschen wahrgenommen werden kann (das Geräusch eines umstürzenden Baums in einem abgelegenen Wald), überhaupt konstituierend für die Bildung von Soundscapes sein kann, stellt sich hier nicht.

Für Urban Public Health ist dieser Ansatz interessant, weil weitere Indizes und Metriken zur Analyse und Systematisierung von Umweltgeräuschen entwickelt wurden, die über die üblichen Lärmindizes bzw. schalldruckpegelbasierten Indizes hinausgehen. Technikfortschritte ermöglichen zunehmend eine umfangreiche Erfassung und Auswertung der großen Datenmengen, die bei der Aufnahme des gesamten Spektrums der akustischen Signale aus der urbanen Umwelt anfallen. Die Daten können u.a. zur visuellen Darstellung der akustischen Umwelt in Form eines Spektrogramms genutzt werden.
In Spektrogrammen werden Frequenz, Amplitude des Schalls und Zeit grafisch dargestellt, die wiederum Grundlage für quantitative Analysen bieten. Bislang sind akustische Metriken ausschließlich für ökologische Fragestellungen angewendet worden, wie z.B. Diversitätsund Gleichmäßigkeitsmetriken oder Metriken, die Beziehungen zwischen geophonen, biophonen und anthrophonen Geräuschquellen beschreiben [55]. In einem ersten, orientierenden Literaturreview konnten insgesamt 23 Soundscape-Indizes identifiziert werden, die sogenannte Alpha- und Betaindizes umfassen [56].

Die Verknüpfung der beiden Soundscape-Ansätze wird das Verständnis der Zusammenhänge zwischen urbaner akustischer Qualität, urbanen räumlichen Zusammenhängen und Effekten auf die menschliche Gesundheit qualitativ und quantitativ deutlich verbessern. Die systematische Anwendung dieser Metriken auf die urbane Geräuschkulisse steht aber noch genauso aus wie die gemeinsame Anwendung der Soundscape-Ansätze in der Lärmwirkungs- bzw. Urban-Public-Health-Forschung.

\section{SALVE - Ein Pilotprojet zu urbaner Klangqualität und Stadtgesundheit}

Vor diesem Hintergrund wurde im Oktober 2018 das von der MERCUR (Mercator Research Center Ruhr) unterstützte zweijährige interdisziplinäre Pilotprojekt SALVE (Acoustic Quality and Health in Urban Environments) in der Metropole Ruhr gestartet. Die beteiligten Disziplinen aus Urban Public Health, Raumplanung und Landschaftsökologie zielen auf:

(i) die Entwicklung messtechnischer und räumlich-statistischer Modelle zur Identifikation von Kriterien für gesundheitsförderliche Klangqualitäten,

(ii) die Erfassung von Wahrnehmungsmustern und Präferenzen der in SALVE erhobenen Soundscapes gemäß der ISO/TS 12913-2:2018 [51] sowie (iii) erste Analysen zu gesundheitlichen Effekten von Soundscapes in stadträumlichen Konstellationen.

Dazu werden umfangreiche Audiodatensätze zu räumlich und zeitlich variierenden Klanglandschaftsdaten im Untersuchungsgebiet von Bochum aufgebaut. Die Analysen erfolgen mit dem erweiterten Metrikenansatz (Widened Array of Metrics, WAM), um ein möglichst breites Verständnis der akustischen Umgebung zu ermöglichen [56]. Der WAM-Ansatz wird ein mehrdimensionales Verständnis von Schall als Signaturen, Cluster und räumliche Disparitäten von Schallphänomenen liefern. Die Idee ist, die Klangheterogenität nicht nur in öffentlichen Räumen, sondern explizit auch im Wohnumfeld der Menschen zu charakterisieren.

Eine ausführliche Beschreibung des Studiendesigns ist in [57] zu finden. Kurz zusammengefasst: Von Februar 2019 bis März 2020 wurden an rund 730 Orten in der Stadt Bochum alle Schallereignisse zu jeweils verschiedenen Tages-, Nacht- und Jahreszeiten gemessen. Die Messorte wurden unter Berücksichtigung unterschiedlicher Landschaftsnutzungstypen zufällig ausgewählt. Hierzu zählen z.B. Wohn- und Mischbebauung, Parks sowie Messorte mit Straßen- und Autobahnnähe. Manuelle Messungen erfolgten durch das SALVE-Team. Pro Messort wurden jeweils 5 min dauernde Schallmessungen durchgeführt. Die manuellen Messungen erfolgen werktags in der Zeit von 9-18 Uhr. Nur bei starkem Regen konnten keine manuellen Messungen durchgeführt werden. Jeder Standort wurde zu allen 4 Jahreszeiten einmal gemessen. Für SALVE stehen zudem 28 automatisierte Aufnahmegeräte zur Verfügung, mit denen an weiteren Messorten jeweils stündlich 23-minütige Schallmessungen durchgeführt wurden - also unabhängig von Tages- und Nachtzeiten sowie Werk- und Feiertagen. Das Design der manuellen und automatisierten Messungen ermöglicht so (i) einen zeitlich begrenzten, aber räumlich detaillierten Sounddatensatz und (ii) eine zeitlich detaillierte, aber räumlich begrenzte Auswahl von longitudinalen Sounddaten über ein Jahr für ausgewählte Landnutzungstypen. Zur Qualitätssiche- 
rung wurden neben dem Studienprotokoll, ein statistischer Analyseplan sowie SOPs (Standard Operation Procedures) erstellt; die Schallmessungen werden mittels Plausibilitätsschecks überprüft. Räumliche Parameter wie die Nähe der Messorte zu Autobahnen, Innenstädten oder die Verfügbarkeit von umliegenden Grünflächen werden per Geoinformationssystem-(GIS-)Analysen einbezogen. Darüber hinaus werden weitere räumliche Datensätze wie Erholungsgebiete, Straßen- und Schienenkorridore, städtische Dichtegradienten, demografische und wirtschaftliche Daten sowie Busund Nahverkehrsstationen in die räumlich-statistischen Analysen einbezogen. Bislang wurden rund $21.300 \mathrm{~h}$ Audioaufnahmen zusammengetragen. Erste Analysen zeigen zum Beispiel, dass Orte mit hohem Grünanteil niedrigere Lärmwerte und Orte mit höheren Lärmwerten und geringeren Grünanteilen einen hohen Arbeitslosenanteil der Bevölkerung aufweisen. Orte mit einem hohen Anteil von biophonen Geräuschquellen (hier: Vögel) liegen erwartungsgemäß nicht nur in den Waldgebieten, sondern auch in Wohngebieten.

SALVE ist somit Ausgangspunkt für einen innovativen Ansatz zum Verständnis der Auswirkungen akustischer Umgebungen auf die städtische öffentliche Gesundheit - über die Lärmminderung hinaus. Die in diesem Projekt gewonnenen Erkenntnisse werden dazu beitragen, lösungsorientierte gesundheitsfördernde Strategien in der Raumplanung zu entwickeln.

\section{Fazit}

Städte sind Lebensräume, in denen Menschen mit ihren städtischen - d.h. technisch-baulichen, sozialen und ökologischen - Umwelten in komplexen Zusammenhängen miteinander interagieren und sich damit gegenseitig beeinflussen. Nachhaltigkeit erfordert zukünftig eine Verdichtung der Städte, die kaum denkbar ist ohne eine Zunahme einer Vielzahl an Geräuschen - meist in Form von Lärm. Um eine Akzep$\operatorname{tanz}$ für die Verdichtung zu erreichen, sind qualitätsvolle, akustisch angenehme städtische Räume erforderlich. Hierfür sind Lärmminderungsmaßnahmen notwendig, aber nicht ausreichend.

Wenn Geräusche nicht nur als Risikofaktor in Form von Lärm, sondern auch als gestaltbare Qualität und wichtige Ressource für eine gesundheitsfördernde Stadt verstanden werden, kann ein vertieftes Verständnis von urbaner Akustik erzielt werden, die weit über laut und leise hinausgeht. Die Verknüpfung der hier vorgestellten SoundscapeAnsätze ermöglicht neue Erkenntnisse, die zur Gestaltung gesundheitsförderlicher urbaner Klangräume beitragen können. Insgesamt geht es um eine Erweiterung der wissenschaftlichen und auch politischen Perspektiven des Konstruktes Lärm. Es geht um die quantitativen und qualitativen Aspekte des Schalls, sowohl in negativer als auch in positiver Hinsicht. Ein umfassenderes Verständnis, wie wir es hier mit den SoundscapeAnsätzen vorschlagen, führt neben der Lärmvermeidung zur einer gesundheitsförderlichen Klanggestaltung, die interdisziplinär erforscht und im Sinne von Health in All Policies (Gesundheit in allen Politikbereichen) umgesetzt werden sollte.

\section{Korrespondenzadresse}

\section{Prof. Dr. Susanne Moebus}

Institut für Urban Public Health, Uni-

versitätsmedizin, Universität Duisburg-

Essen

Hufelandstr 55, 45147 Essen, Deutschland

susanne.moebus@uk-essen.de

Danksagung. Wir danken den Gutachter*innen für hilfreiche Verbesserungsvorschläge. Das SALVEProjekt wird von MERCUR (Mercator Research Centre Ruhr) (Projektnummer Pr-2018-0006) unterstützt.

Funding. Open Access funding provided by Projekt DEAL.

\section{Einhaltung ethischer Richtlinien}

Interessenkonflikt. S. Moebus, D. Gruehn, J. Poppen, R. Sutcliffe, T. Haselhoff und B. Lawrence geben an, dass kein Interessenkonflikt besteht.

Für diesen Beitrag wurden von den Autoren keine Studien an Menschen oder Tieren durchgeführt. Für die aufgeführten Studien gelten die jeweils dort angegebenen ethischen Richtlinien.

Open Access. Dieser Artikel wird unter der Creative Commons Namensnennung 4.0 International Lizenz veröffentlicht, welche die Nutzung, Vervielfältigung, Bearbeitung, Verbreitung und Wiedergabe in jeglichem Medium und Format erlaubt, sofern Sie den/die ursprünglichen Autor(en) und die Quelle ordnungsgemäß nennen, einen Link zur Creative Commons Lizenz beifügen und angeben, ob Änderungen vorgenommen wurden.

Die in diesem Artikel enthaltenen Bilder und sonstiges Drittmaterial unterliegen ebenfalls der genannten Creative Commons Lizenz, sofern sich aus der Abbildungslegende nichts anderes ergibt. Sofern das betreffende Material nicht unter der genannten Creative Commons Lizenz steht und die betreffende Handlung nicht nach gesetzlichen Vorschriften erlaubt ist, ist für die oben aufgeführten Weiterverwendungen des $\mathrm{Ma}$ terials die Einwilligung des jeweiligen Rechteinhabers einzuholen.

Weitere Details zur Lizenz entnehmen Sie bitte der Lizenzinformation auf http://creativecommons.org/ licenses/by/4.0/deed.de.

\section{Literatur}

1. WBGU (2016) Der Umzug der Menschheit: Die transformative KraftderStädte. Wissenschaftlicher Beirat der Bundesregierung Globale Umweltveränderungen (WBGU), Berlin, S89

2. UN-United Nations (2015) Transforming our world: the 2030 agenda for sustainable development. https://sustainabledevelopment.un.org/ content/documents/21252030\%20Agenda $\% 20$ for\%20Sustainable\%20Development \%20web.pdf.Zugegriffen:3.Dez. 2019

3. BartonH, Tsourou C (2013) Healthy urban planning. Routledge, London

4. Babisch W (2003) Stress hormones in the research on cardiovascular effects of noise. Noise Health 5:1-11

5. Lorenz AM (2010) Klangökologie aus sozialwissenschaftlicher Sicht und Chancen für eine klangökologische Rezeptionsforschung. In: Forum Klanglandschaft (Hrsg) Klanglandschaft wörtlich. Akustische Umwelt in transdisziplinärer Perspektive. Forum Klanglandschaft, Akroama Verlag, Basel, S 33-40 (http://www.klanglandschaft.org/ images/documents/klanglandschaft_woertlich_ 2010.pdf.Zugegriffen:21. Februar 2020)

6. European Environment Agency (2014) Noise in Europe 2014. http://www.eea.europa.eu/ publications/noise-in-europe-2014. Zugegriffen: 21. Febr. 2020

7. Fuks K, Moebus S, Hertel et al (2011) Long-term urban particulate air pollution, traffic noise, and arterial blood pressure. Environ Health Perspect 119:1706-17011

8. Barregard L, Bonde E, Ohrstrom E (2009) Risk of hypertension from exposure to road traffic noise in a population-based sample. Occup Environ Med 66:410-415

9. Chang T, Hwang B, Liu C et al (2013) Occupational noise exposure and incident hypertension in men: a prospective cohort study. Am J Epidemiol 177:818-825

10. Selander J, Nilsson ME, Bluhm G et al (2009) Longterm exposure to road traffic noise and myocardial infarction. Epidemiology 20:272-279

11. Babisch W, Beule B, Schust $M$, Kersten N, lsing $H$ (2005) Traffic noise and risk of myocardial infarction. Epidemiology 16:33-40 
12. Sørensen M, Hvidberg M, Andersen ZJ et al (2011) Road traffic noise and stroke: a prospective cohort study. Eur Heart J 32:737-744

13. Kälsch H, Hennig F, Moebus S et al (2014) Are air pollution and traffic noise independently associated with atherosclerosis: the Heinz Nixdorf Recall Study. Eur Heart J 35:853-860

14. Sørensen M, Andersen ZJ, Nordsborg RB et al (2012) Long-term exposure to road traffic noise and incident diabetes: a cohort study. Environ Health Perspect 121:217-222

15. Franzen PL, Buysse DJ (2008) Sleep disturbances and depression: risk relationships for subsequent depression and therapeutic implications. Dialogues Clin Neurosci 10:473-481

16. Halonen Jl, Vahtera J, Stansfeld S et al (2012) Associations between nighttime traffic noise and sleep: the Finnish Public Sector Study. Environ Health Perspect 120:1391-1396

17. Anisman H, Merali Z (2002) Cytokines, stress, and depressive illness. Brain Behav Immun 16:513-524

18. Wager-Smith K, Markou A (2011) Depression: a repair response to stress-induced neuronal microdamage that can grade into a chronic neuroinflammatory condition? Neurosci Biobehav Rev 35:742-764

19. Bundesministerium für Umwelt, Naturschutz und nukleare Sicherheit (2020) Was ist Lärm. https:// www.bmu.de/themen/luft-laerm-verkehr/ laermschutz/laermschutz-im-ueberblick/wasist-laerm/.Zugegriffen:21. Febr. 2020

20. Bundeszentrale für gesundheitliche Aufklärung - BZgA (2008) Lärm und Gesundheit (5.-10.). Bestellnummer 20350000. BMG, Köln

21. Kloepfer M, Griefahn B, Kaniowski AM et al (2006) Leben mit Lärm? Risikobeurteilung und Regulation des Umgebungslärms im Verkehrsbereich. Wissenschaftsethik und Technikfolgenbeurteilung. In: Gethmann (Hrsg) Schriftenreihe der Europäischen Akademie zur Erforschung von Folgen wissenschaftlich-technischer Entwicklungen, Bd. 28. Springer, Berlin Heidelberg New York, S 339

22. Berglund B, Lindvall T, Schwela DH, World Health Organization. Occupational and Environmental Health Team (1999) Guidelines for community noise. World Health Organization. https://www. who.int/docstore/peh/noise/Commnoise2.htm. Zugegriffen: 14.Apr. 2020

23. DIN 1320: 2009-12. Akustik-Begriffe. Beuth Verlag $\mathrm{GmbH}$, Berlin

24. TA Lärm. 6. Allgemeine Verwaltungsvorschrift zum Bundes-Immissionsschutzgesetz (Technische Anleitung zum Schutz gegen Lärm - TA Lärm) Vom 26. August 1998 (GMBI Nr. 26/1998 S. 503).

25. Sächsisches Staatsministerium für Energie, Klimaschutz, Umwelt und Landwirtschaft (2020) Wirkung von Lärm auf den Menschen. https:// www.umwelt.sachsen.de/umwelt/3514.htm. Zugegriffen: 13. Apr. 2020

26. Bundesamt für Justiz (2020) Verordnung zum Schutz der Beschäftigten vor Gefährdungen durch Lärm und Vibrationen (Lärm- und Vibrations-Arbeitsschutzverordnung - LärmVibrationsArbSchV). § 2 Begriffsbestimmungen. Bundesministerium der Justiz und Verbraucherschutz. https://www.gesetze-im-internet.de/l_ rmvibrationsarbschv/_2.html. Zugegriffen: 14 Apr. 2020

27. Bundesministerium für Umwelt, Naturschutz und nukleare Sicherheit (UBA) (2020) Lärmschutz im Überblick. https://www.bmu.de/themen/ luft-laerm-verkehr/laermschutz/laermschutzim-ueberblick/.Zugegriffen: 13.Apr. 2020
28. Payer P (2003) Vom Geräusch zum Lärm. Zur Geschichte des Hörens im 19. und frühen 20. Jahrhundert. In: Aichinger W, Eder FX, Leitner C (Hrsg) Sinne und Erfahrung in der Geschichte. StudienVerlag, Innsbruck, S 173-192

29. Wiktionary. https://de.wiktionary.org/w/index. php?title=Ger\%C3\%A4usch. Zugegriffen: 01. Februar 2020

30. Digitale Wörterbuch der deutschen Sprache. "Geräusch", bereitgestellt durch das Digitale Wörterbuch der deutschen Sprache, https://www. dwds.de/wb/Ger\%C3\%A4usch\#1. Zugegriffen: 21. Jan. 2020

31. Wikipedia. https://de.wikipedia.org/wiki/Ger\%C3 \%A4usch.Zugegriffen: 21. Febr. 2020

32. Ministerium für Wirtschaft, Arbeit und Wohnungsbau (2018) Städtebauliche Lärmfibel. Hinweise für die Bauleitung. Version 2018. https://www. staedtebauliche-laermfibel.de/?p=5\&p2 $=2.2 . \mathrm{Zu}$ gegriffen:21. Febr. 2020

33. Babisch W (2014) Updated exposure-response relationship between road traffic noise and coronary heart diseases: a meta analysis. Noise Health 16:1-9

34. WHO (2018) Environmental Noise Guidelines for the European Region. www.euro.who.int/ en/publications/abstracts/environmental-noiseguidelines-for-the-european-region-2018.Zugegriffen:21. Febr. 2020

35. Guski R (2001) Moderatoren der Lärmwirkungen In:Wichmann HE, Schlipköter HW, FülgraffG (Hrsg) Handbuch der Umweltmedizin, Bd. 7. Ecomed, Landsberg/Lech, S 1-41

36. Forschungsverbund Leiser Verkehr (2013) Bericht zur Situation der Lärmwirkungsforschung in Deutschland. Fokus Verkehrslärm. http://scholar. google.de/scholar_url?url=https $\% 3 \mathrm{~A} \% 2 \mathrm{~F}$ $\% 2$ Fenvironmentalnoise.zeusgmbh.de\%2Fpdfs \%2FBericht_Laermwirkungsforschung_lang. $\mathrm{pdf} \& \mathrm{hl}=\mathrm{de} \& \mathrm{sa}=\mathrm{T} \& \mathrm{i}=\mathrm{ggp} \& \mathrm{ct}=\mathrm{res} \& \mathrm{~cd}=0 \&$ $d=14168065211927416049 \&$ $\mathrm{ei}=\mathrm{jkWbXp2NEs3FmAHF8LKICQ \&}$ scisig=AAGBfm $2 \mathrm{kren} 5 \mathrm{sEAOnwg}$ Q1bCma6XXbTm2A\&nossl=1\&ws=1920x942\& at=Forschungsverbund\%20Leiser\%20Verkehr\& bn=1.Zugegriffen: 25 . Febr. 2020

37. Neuhaus I, Neuhaus F (2016) Akustisch gute Architektur für Straßenräume. Ein Leitfaden für architektonische und städtebauliche Lösungen, die Verkehrslärm reduzieren und mehr Aufenthaltsqualitätschaffen. Publikationsreihe "Chancen im Lärm - Klangraumgestaltung". Basel, Zürich. http://www.cerclebruit.ch/studies/klangraum/ akustisch-gute-architektur_strassenraeume.pdf Zugegriffen:21.Febr. 2020

38. Yang W, Kang J (2005) Acoustic comfort evaluation in urban open public spaces. Appl Acoust 66:211-229

39. Maag T (2016) Urbane Hörräume. In: Sturm U, Bürgin M (Hrsg) Stadtklang. Wege zu einer hörenswerten Stadt. 1 Perspektiven. Vdf Hochschulverlag AG, ETHZürich, Zürich, S26-37

40. Bosshard A, Maag T (2013) Klangraumgestaltung - Chancen im Lärm. Fünf Fallbeispiele im urbanen Raum des Kantons Zürich. Lärminfo 17 der Fachstelle Lärmschutz. Baudirektion Kanton Zürich, Zürich

41. Schweizerischer Bundesrat (2015) Umwelt Schweiz 2015. Bericht des Bundesrates. www.bafu.admin.ch/bafu/de/home/zustand/ publikationen-zum-umweltzustand/umweltschweiz-2015.html.Zugegriffen: 21. Febr. 2020

42. Lercher P, Schulte-Fortkamp B (2013) Soundscape of European cities and landscapes - harmonising.
In: Kang J, Chourmouziadou K, Sakantamis K, Wang B, Hao Y (Hrsg) COST action TD0804 soundscape of European cities and landscapes, oxford (http://soundscape-cost.org/documents/ COST_TD0804_E-book_2013.pdf. Zugegriffen: 10. April 2020.)

43. van Kempen E, Devilee J, Swart W, van Kamp I (2014) Characterizing urban areas with good sound quality: development of a research protocol. Noise Health 16:380-387

44. European Parliament and Council Directive 2002/49/EC of the European Parliament and of the Council of 25th June 2002, relating to the assessment and management of environmental noise (2002). Off.J. Eur. Communities L189:0012-0026.

45. Kang J, Aletta F, Gjestland TT, Brown LA, Botteldooren D, Schulte-Fortkamp B et al (2016) Ten questions on the soundscapes of the built environment. Build Environ 108:284-294

46. Raimbault M, Dubois D (2005) Urban soundscapes: experiences and knowledge. Cities 22:339-350

47. Schafer RM (1977) The soundscape: our sonic environment and the tuning of the world. Destiny Books, Rochester

48. International Organization for Standardization, ISO 12913-1:2014, (2014) Acoustics—Soundscape Part 1-Definition and Conceptual Framework, ISO, Geneva, S3.

49. Kang J, Schulte-Fortkamp B (2016) Soundscape and the built environment. Taylor \& Francis Group LLC, Boca Raton

50. International Organization for Standardization, ISO/TS 12913-2:2018(E) (2018). Acoustics-Soundscape-Part 2: Data collection and reporting requirements, ISO, Geneva.

51. Stack DW, Newman P, Manning RE, Fristrup KM (2010) Reducing visitor noise levels at Muir Woods National Monument using experimental management. J Acoust Soc Am 129:1375-1380

52. Brown AL (2012) A review of progress in soundscapes and an approach to soundscape planning. Int J Acoust Vib 17:73-81

53. Pijanowski BC, Villanueva-Rivera LJ, Dumyahn SL et al (2011) Soundscape ecology: the science of sound in the landscape. BioScience 61:203-216

54. Farina A (2014) Soundscape ecology. Principles, patterns, methods and applications. Springer, Dordrecht

55. Sueur J (2018) Sound analysis and synthesis with R Springer, Cham

56. Lawrence BT, Ahmed S, Sutcliffe R, Poppen J, Moebus S, Gruehn D (2019) A widened array of metrics (WAM) approach to characterizing urban sound environments-the example in SALVE. Proceedings of the 23rd International Congress on Acoustics (ICA), Aachen

57. Sutcliffe R, Lawrence BT, Ahmed S, Gruehn D, Moebus S (2020) Acoustic quality and health in urban environments (SALVE) - a pilot project in the metropolitan Ruhr region, Germany. Cities \& health https://doi.org/10.1080/23748834.2019. 1648362 